\title{
Los activos intangibles y la ventaja competitiva sustentable en las Pymes ecuatorianas
}

\section{Intangible assets and sustainable competitive advantage in Ecuadorian Pymes}

Jadira Monserrath Llerena Gómez. ${ }^{1}$, Mónica Patricia Mayorga Díaz. ${ }^{2}$, Ana Alexandra López Jara. $^{3} \&$ María Alexandra López Paredes. ${ }^{4}$

\begin{abstract}
DOI: https://doi.org/10.33262/visionariodigital.v4i3.1236

The role played by small and medium-sized companies in the contemporary business field has become transcendental anywhere in the world, since these organizations, for the most part, are established with family assets and contribute significantly to the economy of a country, Within its organizational structures, the level of importance of organizational sustainability is revealed, which is why within this research the development of corporate strategic management in the displacement of the leader was identified, and at the same time, the integral financial management of intangible resources is they have transformed into future economic benefits; However, there are a number of difficulties that complicate in some cases their timely assessment, the identification of the drawbacks that exist for the execution of control by organizations regarding the treatment of intangible assets. Therefore, a thorough documentary study on the modern behavior of research edges within the units of analysis was considered essential, so a comparison of economic reports at the global and national level was also made.
\end{abstract}

Keywords: Competitiveness, development, balance, intangibles, sustainability.

\section{Resumen}

El rol desempeñado por las pequeñas y medianas empresas en el campo empresarial contemporáneo se ha convertido en trascendental en cualquier lugar del mundo, una vez

\footnotetext{
${ }^{1}$ Estudiante, Universidad Regional Autónoma de Los Andes, Facultad de Sistemas Mercantiles, Carrera de Contabilidad y Auditoría, monse1251@hotmail.com, ORCID 0000-0002-3902-3117

2 Docente investigadora, Doctora en ciencias económicas, Universidad Regional Autónoma de Los Andes, Facultad de Sistemas Mercantiles, monikmayorgad@gmail.com, ORCID 0000-0003-3071-236X

${ }^{3}$ Docente titular, Magíster en auditoría integral, Universidad Católica de Cuenca, Carrera de Contabilidad y Auditoría, annylopezjara@ hotmail.com, ORCID 0000-0001-6905

${ }^{4}$ Docente investigadora, Doctora en administración y gestión pública, Universidad Técnica de Ambato, Facultad de Ciencias Administrativas, ma.lopez@uta.edu.ec, ORCID 0000-0002-2452-2271
} 
que estas organizaciones en su gran mayoría, se constituyen con patrimonio familiar y contribuyen notablemente a la economía de un país, dentro de sus estructuras organizacionales se revela el nivel de importancia de la sustentabilidad organizacional, razón por la que dentro de esta investigación se identificó el desarrollo del direccionamiento estratégico empresarial en el desplazamiento del líder, así mismo actualmente la gestión financiera integral de los recursos intangibles se han transformado en beneficios económicos futuros; no obstante, existen un sin número de dificultades que complican en algunos casos su valoración oportuna, la identificación de los inconvenientes que existen para la ejecución del control por parte de las organizaciones referentes al tratamiento los activos intangibles. Por consiguiente, se consideró primordial el estudio documental minucioso sobre el comportamiento moderno de las aristas de investigación dentro de las unidades de análisis, por lo que se efectuó además un contraste de los reportes económicos a nivel mundial y nacional en donde se determine el efecto ocasionado en los activos intangibles en la pequeña y mediana empresa y lograr una tropicalización a la realidad empresarial ecuatoriana.

Palabras claves: Competitividad, desarrollo, equilibrio, intangibles, sustentabilidad.

\section{Introducción}

En el contexto del desarrollo de este trabajo se examina el valor de los activos intangibles para convertirse en una ventaja competitiva sustentable, por lo tanto, de acuerdo a Ávila \& Pinkus (2018) se toma a consideración las primordiales magnitudes de la sustentabilidad empresarial, que son los factores económicos, sociales y ambientales, lo que podría ser un desempeño para la PYMES ecuatorianas. Existen nuevas realidades: cambios en el entorno de los negocios, en la fuerza de trabajo, en las expectativas de los clientes y competidores, en las organizaciones, en los gerentes y directores $\mathrm{y}$, de igual manera, la naturaleza del trabajo dentro de las organizaciones está experimentando cambios vertiginosos e intensos, lo que conlleva plantear nuevas maneras de tratar con las personas y administrar las organizaciones (Ruiz, et al., 2018).

\section{PyMES en el Ecuador}

De acuerdo a Carranco (2017) las PyMES son consideradas como un grupo de pequeñas y medianas empresas que, acorde al volumen de ingresos, capital y a la cantidad de trabajadores, además de la magnitud de producción o activos que presentan características propias de este tipo de organizaciones económicas (Delgado \& Chávez, 2018).

Manifiestan Vásquez \& Vergara (2011) en el Ecuador estas organizaciones se dedican a diferentes funciones económicas como industrias manufactureras, transporte, agricultura, el comercio al por mayor y menor de productos, almacenamiento, servicios prestados, bienes inmuebles, construcción, etc. (Sánchez, 2014).

Es necesario señalar que sus diferentes actividades económicas diferencian en los espacios de un país con otro, ya que las medianas, pequeñas y microempresas reciben los mismos elementos, dependerá del nivel de progreso de cada país para poder implantar igualdad o 
desigualdad en las mismas condiciones (Camacho, 2014; Sánchez, 2014; García \& Villafuerte, 2015; Ron \& Sacoto, 2015).

En el contexto internacional, por ejemplo, una organización pequeña en Italia puede ser una gran empresa en Ecuador (Bolaños \& Amanda, 2019).

Las PyMES son organizaciones que se adaptan fácilmente a los cambios generados por la competencia, expresan Delfín \& Acosta (2016), que para ello deben lograr un crecimiento empresarial que se les permita, ideas como progreso económico, dominio empresarial, liderazgo, administración del conocimiento e innovación que es la integración adecuada para el crecimiento para una Pyme.

De acuerdo a la Superintendencia de Compañías Valores y Seguros mediante resolución, nombra la clasificación de pequeñas y medianas organizaciones, PyMES, de acuerdo a la normativa implantada por la Comunidad Andina en su Resolución 1260 y la legislación interna vigente (SUPERCIAS, 2019), corresponden un total de 802.696 de empresas en el Ecuador las cuales son un conjunto de medias y pequeñas empresas, se las determinan a través de las ventas anuales, número de empleados y nivel de producción o sus activos las cuales; según García \& Villafuerte (2015) se clasifican en varias actividades económicas como la agricultura, industrias manufactureras el comercio al por mayor y al por menor, pesca y silvicultura, construcción, transporte, almacenamiento, comunicaciones, bienes inmuebles y servicios prestados a las otras instituciones (Sánchez, 2014), la mayor representación de empresas en el Ecuador según el Instituto Nacional de Estadísticas y Censos (INEC, 2017), es la microempresa y pequeña empresa consideradas PYMES, las cuales tienen mayor incidencia en el país, reporte de medición activos en las PyMES del manejo de intangibles, tiempo de duración (Figura1).

\begin{tabular}{|l|r|r|}
\hline \multicolumn{1}{|c|}{ Tamaño de empresa } & \multicolumn{1}{c|}{$\begin{array}{c}\text { Nro. } \\
\text { Empresas }\end{array}$} & \multicolumn{1}{c|}{$\%$ Total } \\
\hline TOTAL & $\mathbf{8 8 4 . 2 3 6}$ & $\mathbf{1 0 0 , 0 0 \%}$ \\
\hline MICROEMPRESA & 802.696 & $90,78 \%$ \\
\hline PEQUEÑA EMPRESA & 63.814 & $7,22 \%$ \\
\hline MEDIANA EMPRESA "A" & 8.225 & $0,93 \%$ \\
\hline MEDIANA EMPRESA "B" & 5.468 & $0,62 \%$ \\
\hline GRANDE EMPRESA & 4.033 & $0,46 \%$ \\
\hline
\end{tabular}

Figura 1. Empresas según participación nacional (universo DIEE), año 2017 por tamaño de empresa

Fuente: (INEC, 2017)

La información estadística presentada por el Instituto Nacional de Estadísticas y Censos (INEC, 2017), presenta la participación de las empresas, por tamaño de empresa, dando a conocer el número total de las empresas y su porcentaje de participación total. 


\begin{tabular}{|l|c|c|c|c|}
\hline \multicolumn{1}{|c|}{ Variables } & $\begin{array}{c}\text { Micro } \\
\text { Empresa }\end{array}$ & $\begin{array}{c}\text { Pequeña } \\
\text { Empresa }\end{array}$ & $\begin{array}{c}\text { Mediana } \\
\text { Empresa }\end{array}$ & $\begin{array}{c}\text { Grandes } \\
\text { Empresas }\end{array}$ \\
\hline Personal ocupado & De $1-9$ & De $10-49$ & De $50-199$ & $\geq 200$ \\
\hline $\begin{array}{l}\text { Valor bruto de ventas } \\
\text { anuales }\end{array}$ & $\leq 100.000$ & $100.001-1.000 .000$ & $\begin{array}{c}1.000 .001- \\
5.000 .000\end{array}$ & $>5.000 .000$ \\
\hline Monto de activos & Hasta US\$100.000 & $\begin{array}{c}\text { De US\$100.001 } \\
\text { hasta US\$750.000 }\end{array}$ & $\begin{array}{c}\text { De US\$750.001 } \\
\text { hasta US\$ } 3.999 .999\end{array}$ & $\geq 4.000 .000$ \\
\hline
\end{tabular}

Figura2. Clasificación de Pymes

Fuente: (Cámara de Comercio Quito, 2017)

Los intangibles han logrado una notable constancia en los procesos de gestión, dado el efecto que generan en la creación de aprecio a largo plazo. Es así como los diferentes autores reseñan que en el año 1982 los intangibles representaban algo más del 30\% de estimación de mercado de las instituciones, en el año 1992 llegaron a simbolizar un poco más del 60\% y en el año 1997 rebasaron el 80\% del valor del mercado (González, 2015; NIC 38, 1998).

La estimación que han adquirido en los últimos años el capital intelectual y/o activos intangibles en el marco de la sucesión de gestión organizacional, tanto en lo que tiene que ver con el entorno financiero como lo relacionado con la dirección del talento humano y las áreas operativas, viene a señalar que la idea en cuestión va más allá de un término afortunado y pasajero y, por lo opuesto, figura una categoría que responde a una concepción teórica, donde el entendimiento representa el eje central en toda administración exitosa de cualquier empresa (Ramos, 2014).

La clasificación de los activos intangibles, se señala que estos, se pueden juntar en activos intangibles de recursos humanos, de empresa interna y de estructura externa: Activos Intangibles de Recursos Humanos, según se describen a las aptitudes y conocimiento de los recursos humanos de las organizaciones. Activos Intangibles de Estructura Interna, como la capacidad de los sistemas de información de que dispone la empresa; o procesos, los activos intangibles de Estructura Externa, como la clientela o las marcas, la visión e interés de los activos intangibles viene sobre todo por la nueva magnitud empresarial que da mayor valor a lo inmaterial sobre lo productivo (Carrillo \& Tato, 2004)

Como lo explica la NIC 38 (1998) \& Bertolino et al. (2008) la descripción de un activo intangible pretende que éste sea identificable para poderlo diferenciar de la plusvalía, la cual es reconocida en una mezcla de negocios, es un activo que representa los futuros beneficios económicos que se manifiestan de otros activos adquiridos en una combinación de negocios que no están determinados individualmente y reconocidos de modo separado. Los beneficios económicos futuros pueden provenir de sinergias entre los activos identificables adquiridos o de activos que, individualmente, no ejecutan la naturaleza para su reconocimiento en los estados financieros (Norma Internacional de Contabilidad 38,2012).

Un activo es identificable argumentan Mite, et al. (2016) 
(a) es separable, es decir, es susceptible de ser separado o escindido de la entidad y vendido, transferido, dado en explotación, arrendado o intercambiado, ya sea individualmente o junto con un contrato, activo identificable o pasivo con los que guarde relación, independientemente de que la entidad tenga la intención de llevar a cabo la separación

(b) surge de derechos contractuales o de otros derechos legales, con independencia de que esos derechos sean transferibles o separables de la entidad o de otros derechos $\mathrm{u}$ obligaciones (NIC 38, 1998).

Según la NIIF 3 (2007), Cifuentes (2012) \& Palau (2017) la finalidad de esta NIIF consiste en detallar la información financiera a manifestar por una entidad cuando lleve a cabo una unión de negocios, en particular, especifica que todas las combinaciones de negocios se contabilizarán asignando el método de adquisición; en función del mismo, la entidad adquirente reconocerá los activos, pasivos y pasivos contingentes identificables de la entidad adquirida por sus valores razonables, en la fecha de adquisición y también reconocerá el fondo de comercio, que se someterá a pruebas para detectar cualquier deterioro de su valor, en vez de amortizarse, para alcanzarlo (Cifuentes ,2012), esta NIIF establece principios y requerimientos sobre la forma en que la entidad adquirente (NIIF 3, 2007):

(a) reconocerá y medirá en sus estados financieros los activos identificables adquiridos, los pasivos asumidos y cualquier participación no controladora en la entidad adquirida

(b) reconocerá y medirá la plusvalía adquirida en la combinación de negocios o una ganancia procedente de una compra en condiciones muy ventajosas

y (c) determinará qué información revelará para permitir que los usuarios de los estados financieros evalúen la naturaleza y los efectos financieros de la combinación de negocios (NIIF 3, 2007; Flores, 2018; Orozco, 2018).

En tal sentido, es menester determinar la incidencia de estas organizaciones en los diferentes informes a nivel mundial y nacional, considerando a los activos intangibles y ventaja competitiva como análisis de estudio.

\section{Materiales y Método}

Una revisión documental rigurosa enfocada a la sustentabilidad empresarial y activos intangibles permitió definir la incidencia entre las aristas de investigadas en los diferentes informes a nivel mundial y nacional; asimismo la visión documental permite precisar las investigaciones elaboradas anteriormente, las autorías y sus discusiones; diseñar el objeto de estudio; componer premisas de partida, fijar autores para preparar una base teórica; formar relaciones entre trabajos; indagar preguntas y objetivos de investigación; ejecutar las estéticas de los procedimientos metodologías de abordaje; implantar semejanzas y diferencias entre los trabajos y las ideas del investigador, tipificar experiencias; resaltar los elementos más abordados con sus esquemas observaciones y determinar los ámbitos no explorados (Acevedo, 2018; Suárez, et al., 2018 ; Valencia, 2012). 


\section{Activos intangibles}

Como identifica Julve (2000) Los activos intangibles más difíciles de cuantificar debido a las diferentes fases de su ciclo de vida, a la conducta de los consumidores y a la visión de problemas o confusiones imprevistas. Actualmente según González (2015), los activos intangibles son los responsables en un eminente porcentaje de la creación de significación en las organizaciones, aunque, la gran duda que surge es cómo cuantificarlos, pues ya se ha hecho famosa la frase de que lo que no se puede calcular no se puede administrar, de tal manera que puedan ser reconocidos absolutamente con la creación de valor, tal como se puede ejecutar con los activos tangibles, ya que su efecto la generación de ventaja competitiva y en la creación de valor puede ser comprobada de manera directa en los estados financieros de las empresas (Carrillo \& Tato , 2004; León \& Cifuentes, 2009; Romero \& Zilber, 2015) (Figura 3).

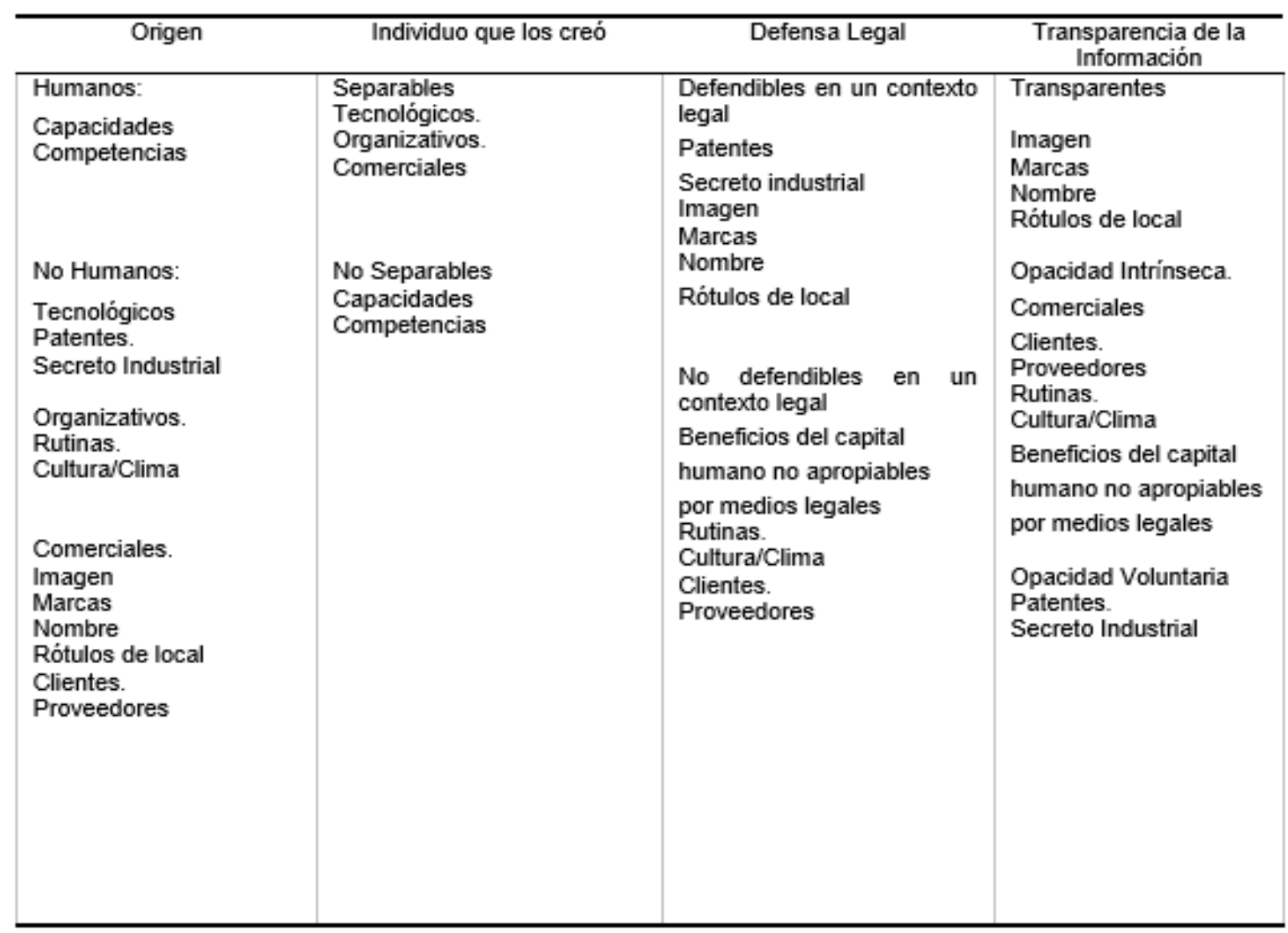

Figura3. Clasificación de los Activos Intangibles

Fuente: Ramos (2014)

\section{Sustentabilidad}

La sustentabilidad empresarial se confronta cara a cara al modelo de desarrollo y a las inflexiones que difunde el dominio de la ética ecológica. Son diferentes éticas que se encaran unas con otras y el dilema ético arduo de resolver es si aceptamos o no manejar estos recursos 
naturales para suministrar la matriz energética que alimenta el funcionamiento de nuestros hogares e industrias se puede decir que de esta manera se maneja una sustentabilidad ecológica (Suárez, 2013), la cual consiste en acoger estrategias de negocio para cubrir las necesidades de la empresa y sus interesados, mientras se dirige y sustenta a los recursos naturales que serán necesarios en el futuro y se apoya en el desarrollo de la sociedad (Ávila \& Pinkus , 2018), de acuerdo con el logro de cualquier proyecto se precisa a partir de la habilidad que tenga el decisor para modificar dichas alteraciones en ventajas (Ávila \& Pinkus , 2018 ; Barbosa , 2016 ; Carro, et al., 2017; Zulueta, et al., 2013) (Figura 4).

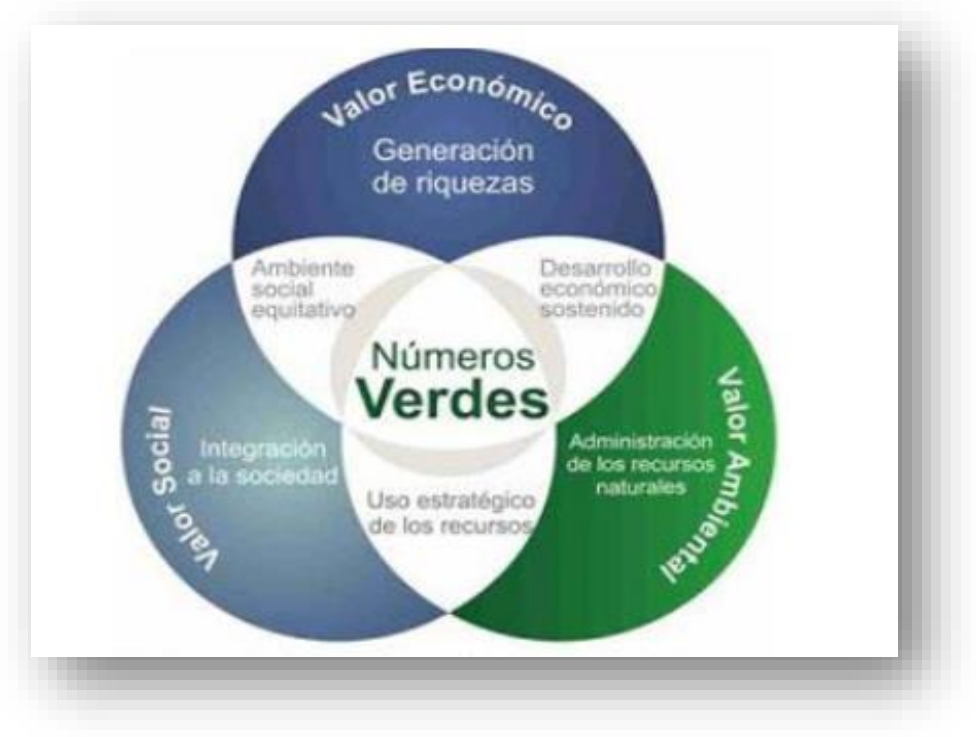

Figura 4. Modelo de Desarrollo Sustentable

Fuente: Zulueta, et al. (2013)

Los tipos de negocio pueden ser observados, desde la perspectiva de la sustentabilidad, en tres dimensiones que en conclusión puedan transformarse en una sola: sustentabilidad económica, ambiental y social (Ávila \& Pinkus, 2018).

\section{Activos intangibles en las PyMES}

Desde la perspectiva de Simarro, et al. (2013) la rápida variación socio-económica de las últimas décadas hacia una entidad basada en el entendimiento, ha hecho que los intangibles estén cada vez más relacionados con el transcurso de creación de valor dentro de las instituciones; en este nuevo entorno, los entes deben ser capaces de determinar y medir sus activos intangibles (Simarro, et al., 2013). Esto incluye compenetrarse con los diversos problemas, entre los que se remarcan son los instrumentos generalmente aceptados para poderlos medir, así mismo el desconocimiento de su conceptualización disponible para comprenderlos y señalarlos (Simarro, et al. 2013). Destacan (Quijano, et al., 2017; Simarro, et al., 2013) dado que los servicios de las organizaciones incluyen destacados elementos inmateriales, su gestión puede aprovechar ampliamente con la identificación y medición de los 
activos intangibles generadores de valor de tal forma como la innovación, el conocimiento, los recursos humanos, la conexión con clientes, proveedores y competidores.

Argumenta Simarro, et al. (2013) \& Huamaní (2002) los intangibles son el efecto de la incorporación de del conocimiento e información de las actividades productivas que desempeñan varias empresas, al poseer el conocimiento de estas actividades es poder desarrollar ventajas competitivas en el entorno organizacional, puesto que las actividades tanto como los recursos de los activos intangibles que los reúnen son manifestaciones del conocimiento (Simarro, et al., 2013).

Se concluye entonces que los activos intangibles corresponden a bienes, impuesto que son controlados económicamente por el ente resultante de biografía pasados, de los que se pretende que la empresa obtenga ingresos o rendimientos económicos en la posibilidad; no obstante, para su recepción, los activos intangibles no sólo deben actuar con la explicación frontal, sino que, todavía, como ya ha quedado determinado, es precisado que sean identificables. Se asume de un concepto nuevo, el cual se desconoce en nuestra información contable, aceptablemente su separabilidad, es hablar, la expectativa de que pueda ser arrendado, vendido, intercambiado, entre otros (Tabla 1).

Tabla 1. Base General

\section{Activos intangibles:}

La práctica general en el País, era registrar las marcas, patentes, derechos de llave, gastos de Las NIIF no permiten registrar como organización y otros activos activo los gastos de organización, intangibles a su valor de costo y sino como gastos.

amortizarlos hasta un máximo de 10 años.

\section{NIC 38}

TEMA 18
De los costos de investigación y desarrollo, las NIIF permiten reconocer como activo únicamente los incurridos en la etapa de desarrollo. Los costos de investigación deben registrarse como gastos del período.

NIIF 3

Las marcas, derecho mercantil y otros intangibles deberán valuarse a su valor actual, aplicando técnicas de proyección de beneficios económicos futuros y descontándolos a valor presente a una tasa de costo relevante para la entidad (Parker Randall Guatemala,2010).
La NIIF PYMES únicamente reconoce el método de costo para valorar los intangibles, ya no se permite el modelo de revaluación.

Se establece una vida finita para amortizar. Cuando no se puede determinar la vida útil se asume una vida útil máxima de 10 años para todos los intangibles (Parker Randall Guatemala,2010).

Elaboración: propia a partir de bibliografía consultada 
Por consiguiente, la sustentabilidad se tratará como un componente importante que puede lograr la expansión económica deseada por las organizaciones que conforman las PyMES, la cual busca renombre en diferente materialidad, como el confort de sus empleados, productos o servicios de calidad, además del impacto social, económico, político, ambiental, y también fomentar el crecimiento tanto económico como social de un país.

\section{Resultados y discusión}

\section{Plan sustentabilidad en las PyMES}

Sostiene Rovira \& Hiriart (2015) la importancia de las dimensiones del desarrollo sustentable; para que América Latina y el Caribe logre alcanzar las prioridades a establecer en la futura agenda de desarrollo después el 2015, se requerirá de una gran movilización de recursos en las dimensiones del incremento sustentable (económica, social y ambiental).Los objetivos de desarrollo sustentable serán una herramienta para poner en práctica y monitorear la agenda para el desarrollo después del 2015, incluyendo obligaciones, responsabilidades y oportunidades para otros países. Consideran Macías, et al. (2015 ) a pesar de la importancia de dichas empresas para la economía de un país, poco se ha realizado para concientizar a sus líderes para que tomen acciones pertinentes, evitando en lo posible futuros conflictos para acceder a los recursos necesarios; sino por el contrario que alcancen la sustentabilidad organizacional, de acuerdo a lo que se ha considerado como las principales dimensiones de la sustentabilidad organizacional, que son los factores sociales, ambientales y económicos. Tal conceptualización está plenamente calificada para incorporar lo necesario para evaluar los impactos de esas tres dimensiones, adoptándose una frase popular y común en el ambiente empresarial (Ávila \& Pinkus, 2018; López, 2014).

Expresan (Velázquez \& Vargas, 2013 ; Gali, 2013; Ramírez, 2003) que actualmente las empresas tienen día tras día que sobrevivir debido a las crecientes necesidades materiales, la escasez de recursos naturales y las desigualdades, la sustentabilidad toma un papel clave como estrategia global, basada en la prosperidad económica, el balance ecológico y el bien común, la globalización en los negocios ha vuelto el panorama más complejo; el aumento de competidores, el acceso a nuevos mercados y el desarrollo de nuevas tecnologías han llevado a los negocios a centrar esfuerzos en construir una identidad que fortalezca sus competencias (Velázquez \& Vargas, 2013), estas fortalezas se basan en la ventaja competitiva, la cual existe cuando hay una equivalencia de las competencias distintivas de una empresa, por lo cual las necesidades particulares del cliente permiten generar una ventaja competitiva (Velázquez \& Vargas, 2013; Ramírez, 2003). Deduce Montenegro (2016) la sustentabilidad financiera en las organizaciones está dirigida a satisfacer los requerimientos económicos actuales de la empresa, pero sin sacrificar las capacidades futuras de la organización y las generaciones siguientes al buscar satisfacer sus propias necesidades; es decir, se debe buscar el perfecto equilibrio entre estos dos elementos. Señala Álvarez \& Vargas (2012) generalmente la industria, está inmersa en un medio social y ambiental; por lo que, la intranquilidad de las organizaciones de detectar un equilibrio que permita el suministrar y lucrarse del sistema es verdaderamente fundamental (Velázquez \& Vargas, 2013). 
Refieren Velázquez \& Vargas, (2013), Álvarez \& Vargas (2012) \& Alier (1994) la sustentabilidad está encaminada para ubicar el equilibrio económico, ecológico y social, dando como resultado la prosperidad y la capitalización de recursos, en la teoría como recursos y capacidades, la idea de sustentabilidad competitiva, se refiere al equilibrio, ya que las empresas pueden examinar a la competencia en función de beneficios excepcionales (Velázquez \& Vargas, 2013). Mencionan Álvarez \& Vargas, (2012) \& Alier (1994) en un ambiente actual, enormemente competitivo y englobado, es necesario el estudio de la sustentabilidad, teniendo en cuenta que entre los agentes competitivos sólo hay un ganador y muchos perdedores, omitiendo los factores sociales y ambientales, contradictorio a lo que plantea la sustentabilidad, una estrategia de la sustentabilidades asegurar un logro permanente en el tiempo. (Velázquez \& Vargas, 2013).

Ratifican Velázquez \& Vargas (2013), Solleiro \& Castañón (2005), Montoya, et al. (2008) que la competitividad es la competencia de una empresa para conservar su aportación lucrativa en la industria, se genera en nuevas estrategias organizacionales, en el incremento de la productividad, en la capacidad empresarial para colaborar en negociaciones con instituciones y empresas del mercado, y en la realidad de un ambiente competitivo definido por el mundo empresarial (Viteri, 2015). Afirma Rojas (2017) que el tratamiento de los intangibles en las organizaciones es un factor que impacta su componente administrativo e influye en el desempeño si se considera al ser humano como eje central en el entorno organizacional. Por lo tanto, las empresas siguen avanzando en estudios y metodologías para determinar la influencia que intangibles como el capital social.

Señalan González, et al. (2016) que la sustentabilidad de las organizaciones a largo plazo es una problemática que dispone de abundante y plural literatura, muestra enfoques más allá de la sustentabilidad ambiental, incorporando como la legitimidad social como otra dimensión.

De cara a la realidad empresarial moderna, los entes mencionados tienen que sobrevivir a las diferentes necesidades crecientes en este caso materiales, la insuficiencia de bienes naturales, por lo que, la sustentabilidad se transforma en un elemento fundamental, para la determinación de una estrategia que engloba a la prosperidad económica, un balance ecológico y además del bien común; de esta manera las PYMES pueden optimizar sus activos intangibles como una ventaja sustentable competitiva.

Para Haigh (2017) \& Brand Finance (2020) las empresas necesitan prestar mayor atención a la posible clasificación y la vida útil de los activos intangibles identificables en el negocio de la compañía adquirida, ya que impactará directamente en las ganancias futuras del grupo adquiriente. Por lo tanto muchas de las organizaciones no dan valor a estos intangibles siendo muy importantes de tomar en cuenta por ejemplo las marcas son unos de los activos intangibles más importantes con una vida útil indefinida, manifiestan Haigh (2017), Brand Finance (2020) es importante establecer un modelo de valoración consistente que aplique las mejores técnicas de valoración en cada comprobación anual de deterioro del valor (Haigh, 2017; Brand Finance, 2020), por consiguiente los porcentajes establecidos desde el año 2001 han variado 
considerablemente hasta el siguiente análisis que es en el año 2016 donde se admira más énfasis en la evaluación de los activos intangibles en las organizaciones (Tabla 2).

Tabla 2. Grado de importancia de los activos intangibles.

\begin{tabular}{|c|c|c|c|}
\hline & 2001 & 2016 & 2016 \\
\hline & Analistas & Analistas & CFOs \\
\hline \multicolumn{4}{|l|}{ Coinciden en que las marcas son más importantes para... } \\
\hline ... gestionar riesgos & $20 \%$ & $53 \%$ & $68 \%$ \\
\hline ... tomar decisiones crediticias & $18 \%$ & $47 \%$ & $53 \%$ \\
\hline ... la planificación fiscal & $4 \%$ & $25 \%$ & $27 \%$ \\
\hline ... fusiones $\mathrm{y}$ adquisiciones & $53 \%$ & $72 \%$ & $76 \%$ \\
\hline ... el reporting financiero & $22 \%$ & $38 \%$ & $53 \%$ \\
\hline ... los sectores que no suelen tener marcas & $52 \%$ & $53 \%$ & $53 \%$ \\
\hline Las marcas globales van a desplazar inevitablemente a las marcas locales & $41 \%$ & $34 \%$ & $32 \%$ \\
\hline $\begin{array}{l}\text { Las marcas de mercados emergentes van a desplazar inevitablemente a las marcas } \\
\text { globales }\end{array}$ & & $16 \%$ & $16 \%$ \\
\hline $\begin{array}{l}\text { Todos los intangibles adquiridos deberian incluirse en el balance financiero de } \\
\text { manera separada }\end{array}$ & & $79 \%$ & $80 \%$ \\
\hline $\begin{array}{l}\text { Todos las marcas generadas internamente deberían incluirse en el balance } \\
\text { financiero de manera separada }\end{array}$ & $56 \%$ & $68 \%$ & $58 \%$ \\
\hline Todos los intangibles deberian revaluarse anualmente & & $73 \%$ & $58 \%$ \\
\hline \multicolumn{4}{|l|}{ ¿Quíén deberia preparar las valoraciones de los intangibles incluidos en las cuentas anuales? } \\
\hline Evaluadores de intangibles independientes y externos & & $58 \%$ & $46 \%$ \\
\hline Evaluadores de intangibles que trabajen para los auditores de la empresa & & $29 \%$ & $19 \%$ \\
\hline Empleados y directivos de las companías implicadas & & $11 \%$ & $30 \%$ \\
\hline Otros & & $2 \%$ & $5 \%$ \\
\hline
\end{tabular}

Fuente: Haigh (2017)

Argumentan Haigh (2017) \& Brand Finance (2020) en América Latina, Argentina se ha considerado el país más intangible, seguido por México y España; sin embargo, en Argentina, el valor intangible declarado representa únicamente el $3 \%$ del valor total de la empresa. Se trata de la menor proporción de todos los países analizados, lo que indica un reporting de menor calidad, provocado mayormente por la falta de fusiones y adquisiciones en la región. La proporción en España es la más alta, encontrándose en el $20 \%$, seguido de Brasil (15\%), Colombia (15\%) y México (13 \%) (Figura5) (Haigh, 2017; Brand Finance, 2020).

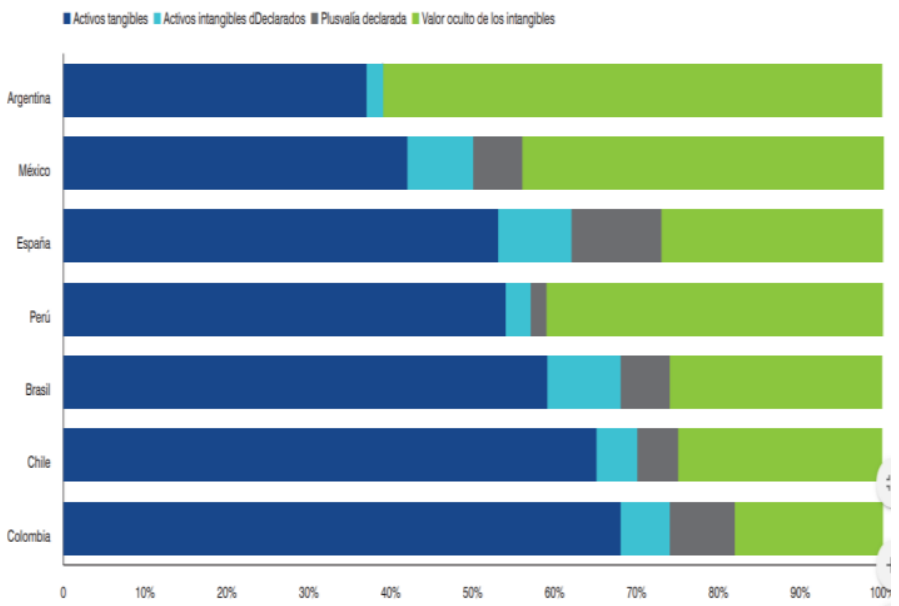

Figura 5. Países más intangibles España y América Latina

Fuente: Haigh (2017) 
El valor intangible declarado (incluyendo la plusvalía) del sector bancario asciende a 40 mil millones de dólares, lo que constituye un $8 \%$ del total de 497 mil millones de valor empresarial; aunque la proporción es mucho menor que el $18 \%$ de los bancos españoles, el ratio se encuentra alineado con el $8 \%$ del sector global; los sectores del petróleo y el gas, la minería y la venta al por menor son los que menor proporción de valor de intangibles declarados presentan en relación al valor de negocio general: $3 \%, 4 \%$ y $9 \%$ respectivamente (Haigh, 2017; Brand Finance, 2020) .En el sector de venta al por menor, una proporción significativa del valor intangible total la constituyen los intangibles relacionados con el consumidor, que no aparecen totalmente reflejados en los balances financieros de los países de la región (Haigh, 2017; Brand Finance, 2020) (Figura 6).

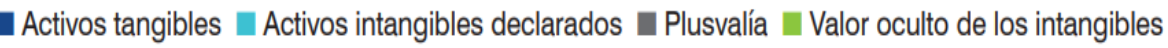

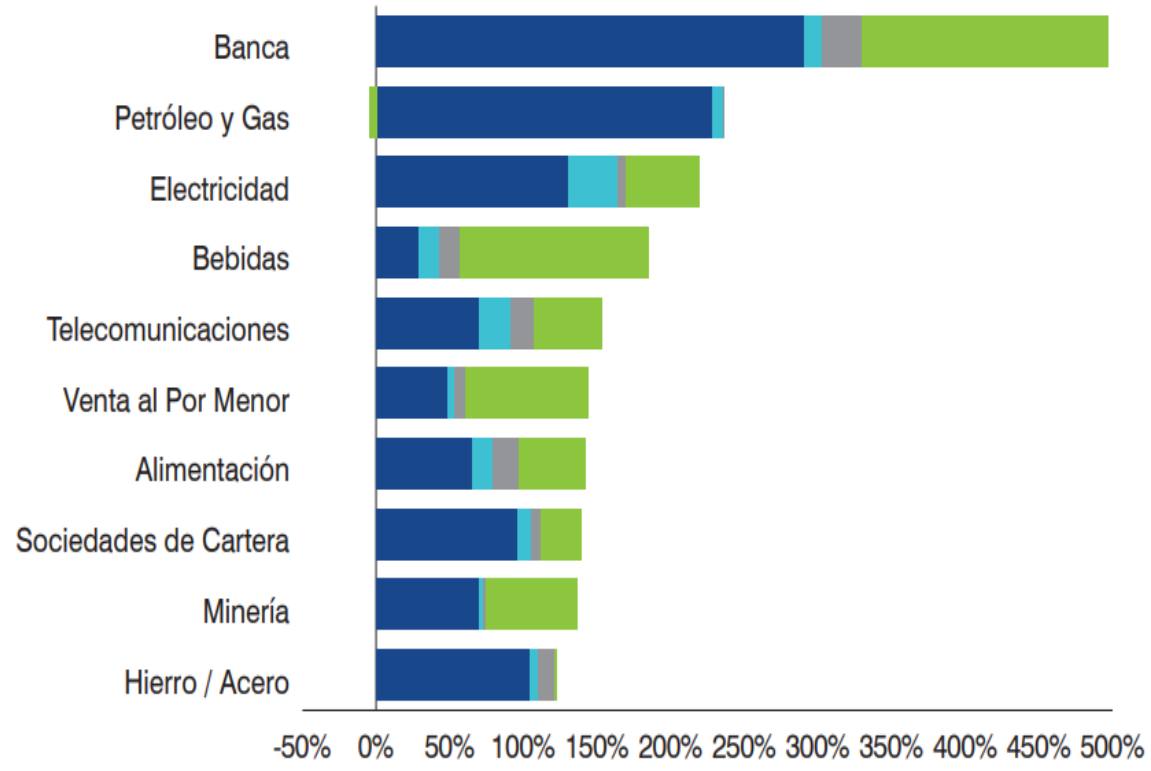

Figura 6. Sectores según su valor empresarial en América Latina

Fuente: Haigh (2017)

Declara el Instituto Ecuatoriano de Propiedad Intelectual (IEPI, 2019) el manual tiene por fin prestar asistencia a los Estados miembros para desarrollar, fomentar y fortalecer la capacidad de las instituciones nacionales de propiedad intelectual (P.I.) mediante el desarrollo de infraestructura y de otros servicios destinados a mejorar la funcionalidad de las instituciones nacionales de propiedad intelectual (P.I.) y promover un equilibrio adecuado entre la protección de la (P.I.) y el interés público (Organización Mundial de Propiedad Intelectual (OMPI), 2016).

La revisión documental permitió reconocer que en el Ecuador existe el manual de valoración de los intangibles tiene como objetivo proporciona un examen de varias metodologías de valoración y ofrece orientación para la evaluación rápida y sistémica de nuevas tecnologías, 
ofrecer respuestas a los problemas que se encuentran en el ámbito en el que hace falta hallar soluciones de manera rápida y práctica (Tabla 3).

Tabla 3. Manual de valoración de activos intangibles de propiedad intelectual.

\begin{tabular}{|c|c|c|}
\hline REPORTE & DESCRIPCIÓN & ANÁLISIS O CRÍTICA \\
\hline $\begin{array}{ll}\text { Manual } & \text { de } \\
\text { valoración } & \text { de } \\
\text { activos } & \\
\text { intangibles } & \text { de } \\
\text { propiedad } & \\
\text { intelectual. } & \end{array}$ & $\begin{array}{l}\text { Proporciona asesoramiento práctico para prestar } \\
\text { asistencia a instituciones de investigación } \\
\text { financiados con fondos del estado con el fin de: } \\
\text { a) Reconocer sus valiosos activos intangibles; } \\
\text { b) Clasificar esos activos usando distintos } \\
\text { métodos de valoración, cualitativos y } \\
\text { cuantitativos; } \\
\text { c) Gestionar los activos que han sido } \\
\text { considerados valiosos para las colaboraciones y } \\
\text { mercados estratégicos; } \\
\text { d) Comercializar los activos intangibles que } \\
\text { puedan tener valor de mercado }\end{array}$ & $\begin{array}{l}\text { Este manual contiene estudios } \\
\text { de casos, muchos de los cuales } \\
\text { tienen su origen en casos } \\
\text { reales, y se ofrecen ejemplos } \\
\text { prácticos que explican la } \\
\text { manera en que la valoración de } \\
\text { los activos intangibles puede } \\
\text { proporcionar una base sólida } \\
\text { para adoptar decisiones } \\
\text { estratégicas de gestión de } \\
\text { dichos activos. }\end{array}$ \\
\hline
\end{tabular}

Elaboración propia a partir de bibliografía consultada

\section{Conclusiones.}

1. Las estadísticas descritas por Haigh (2017) en América Latina muestran la incidencia que tienen los activos intangibles en organizaciones de distinta naturaleza sin dejar a un lado la plusvalía, además la sustentabilidad que tienen los sectores empresariales con mayor declaración de sus intangibles, esto como ventaja competitiva frente a otros sectores que no hacen énfasis en el diagnóstico de sus activos intangibles.

2. Algunas organizaciones en el Ecuador carecen estudios de la medición del grado de valorización de los intangibles, siendo esto estadísticamente comprobado, que en muchos de los sectores empresariales de otros países latinos es una ventaja sustentable competitiva lo que hace que reconozcan como valiosos a sus intangibles en muchas de sus organizaciones.

3. El Ecuador cuenta con un manual de valoración de los activos intangibles de la propiedad intelectual, contiene metodologías básicas para su valoración lo que no garantiza la sustentabilidad de las organizaciones, en vista de que no se está creando una ventaja competitiva entre países latinoamericanos ni muchos menos entre empresas del mismo sector dentro del país.

4. El análisis consciente del nivel de valoración de los activos intangibles implica la generación de una línea de base sólida de aspectos financieros y económicos, para la toma de decisiones gerenciales en cualquier tipo de organización. 


\section{Referencias Bibliográficas}

Acevedo, M. (2018). Participación ciudadana, medio de legitimación de lo público (Doctoral dissertation, Corporación Universitaria Minuto de Dios).

Alier, J. M. (1994). De la economía ecológica al ecologismo popular (Vol. 60). Icaria Editorial.

Álvarez, L. V. V., \& Vargas-Hernández, J. G. (2012). La sustentabilidad como modelo de desarrollo responsable y competitivo. Ingeniería de Recursos Naturales y del Ambiente, (11), 97-107.

Ávila, C. M., \& Pinkus, M. J. (2018). Teorías económico-ambientales y su vínculo con la dimensión social de la sustentabilidad en Áreas Naturales Protegidas. CienciaUAT, 13(1), 108-122.

Barbosa, J. (2016). Modelo de evaluación de pequeños proyectos en OCENSA (Master's thesis, Universidad EAFIT).

Bertolino, G., Díaz, T., \& Suardi, D. (2008). Los activos intangibles y la Contabilidad. In III Jornadas Universitarias Internacionales de Contabilidad: Montevideo, Uruguay, 5, 6 y 7 de noviembre de 2008 (p. 7).

Bolaños, P., \& Amanda, C. (2019). Actualidad de la gestión empresarial en las pymes (Current Business Management in SMEs). Apuntes Contables, (24).

Brand Finance. (2020). Rankings and Reports. Obtenido de: https://brandfinance.com/.

Camacho, N. E. (2014). Responsabilidad social empresarial, RSE en las Pymes: estudio en el Distrito Metropolitano de Quito (Master's thesis, Universidad Andina Simón Bolívar, Sede Ecuador).

Cámara de Comercio Quito. (2017). Boletín Jurídico. Obtenido de : http://www.ccq.ec/wpcontent/uploads/2017/06/Consulta_Societaria_Junio_2017.pdf.

Carranco, R. (2017). La aportación de las pequeñas y medianas empresas (PYMES) en la economía ecuatoriana. Universidad Veracruzana.

Carrillo, M. V., \& Tato, J. L. (2004). La nueva dimensión de comunicación empresarial en el entorno de los activos intangibles. La comunicación espiral. Razón y palabra, 39.

Carro, J., Sarmiento, S., \& Rosano, G. (2017). La cultura organizacional y su influencia en la sustentabilidad empresarial. La importancia de la cultura en la sustentabilidad empresarial. Estudios Gerenciales, 33(145), 352-365.

Cifuentes, J. A. (2012). Análisis de la Norma Internacional de Información Financiera número tres combinaciones de negocios (NIIF 3) con referencia a las Normas Internacionales de Contabilidad NIC 24 (Información a revelar sobre partes vinculadas), NIC 27 (Estados finacieros consolidados y separados), NIC 28 (Inversiones en entidades 
asociadas), NIC 31 (Participaciones en negocios conjuntos) y sus implementación en las empresas (Bachelor's thesis).

Delfín, F. L., \& Acosta, M. P. (2016). Importancia y análisis del desarrollo empresarial. Revista científica Pensamiento y gestión, (40).

Delgado, D., \& Chávez, G. (2018). Las Pymes en el Ecuador. Observatorio de la economía latinoamericana.

Flores, L. (2018). Análisis de adquisición por etapas en una combinación de negocios bajo NIIF 3 (Doctoral dissertation, Universidad de Concepción).

Gali, J. M. (2013). Marketing de sostenibilidad. Profit Editorial.

García, G. H., \& Villafuerte, M. F. (2015). Las restricciones al financiamiento de las PYMES del Ecuador y su incidencia en la política de inversiones. Actualidad contable FACES, 18(30), 49-73.

González, A., Mandirola, N., \& Miles, J. (2016). Sustentabilidad: liderar organizaciones migrantes en el Uruguay-Aspectos asociados al alto desempeño empresarial. Journal of technology management \& innovation, 11(1), 55-64.

González, P. (2015). Propuesta de un modelo para medir activos intangibles en empresas de software a partir de una herramienta multicriterio. Estudios Gerenciales, 31(135), 191201.

Haigh, D. (2017). Informe anual del valor de los intangibles en todo el mundo. Recuperado $d e:$

file:///C:/Users/Usuario/Desktop/gift_report_2017_ce_version_high_res_version.pdf.

Huamaní, P. (2002). Gestión del conocimiento: Un nuevo paradigma organizacional. Gestión en el Tercer Milenio, 5(9).

Instituto Ecuatoriano de la Propiedad Intelectua (IEPI). (2019). La propiedad intelectual vinculada al turismo $y \quad a$ la cultura. Obtenido de: https://www.derechosintelectuales.gob.ec/wpcontent/uploads/2019/09/PITURISMO/i ndex.html.

Instituto Nacional de Estadística y Censos (INEC). (2017). Directorio de Empresas y Establecimientos $2017 . \quad$ Obtenido de: https://www.ecuadorencifras.gob.ec/documentos/webinec/Estadisticas_Economicas/D irectorioEmpresas/Directorio_Empresas_2017/Documentos_DIEE_2017/Documentos _DIEE_2017/Principales_Resultados_DIEE_2017.pdf.

Julve, J. G. (2000). Implicaciones del Capital Social para la Ventaja Competitiva de la Empresa en un Contexto Evolutivo. España: Universitat Jauum I. 
León, I. M., \& Cifuentes, I. O. (2009). La medición de la reputación empresarial: problemática y propuesta. Investigaciones europeas de dirección y economía de la empresa, 15(2), 127-142.

López, J. (2014). Fuentes de financiamiento para las empresas. Obtenido de: https://www.Gestiopolis.com/fuentes-de-financiamiento-para-las empresas.

Macías, C. G., Hernández, I. Z., \& Salgado, P. M. (2015). Sustentabilidad organizacional en pymes familiares restauranteras de La Jonquera en Cataluña, España. Nóesis. Revista de Ciencias Sociales y Humanidades, 24(47-1), 80-97.

Mite, G., Narcisa, D., \& Parra Flores, N. A. (2016). Análisis de los métodos de valoración de activos intangibles (marcas), de una empresa comercial.

Montenegro, H. J. (2016). Diseño de un plan estratégico de negocios que permita la sustentabilidad financiera de las PyMES. Caso estudio: Red Todo Artesano CA, Municipio Valencia Edo. Carabobo (Master's thesis).

Montoya, L. A., Montoya, I. A., \& Castellanos, O. F. (2008). De la noción de competitividad a las ventajas de la integración empresarial. Revista de la Facultad de Ciencias Económicas: Investigación y Reflexión, 16(1), 59-70.

Norma Internacional de Contabilidad 38 (NIC 38). (2012). Activos intangibles. Obtenido de: https://www.mef.gob.pe/contenidos/conta_publ/con_nor_co/vigentes/nic/NIC_038_20 14.pdf.

Norma Internacional de Información Financiera 3 (NIIF 3) (2007). Combinaciones de negocios. Recuperado de: http://www.normasinternacionalesdecontabilidad.es/nic/pdf/niif3.pdf.

Organización Mundial de la Propiedad Intelectual (OMPI). (2016). Guía práctica para la valoración de los activos intangibles en las instituciones de investigación y desarrollo. Obtenido de: https://www.wipo.int/edocs/mdocs/mdocs/es/cdip_17/cdip_17_inf_2.pdf.

Orozco, M. (2018). Evolución de las Normas Internacionales de Información Financiera y su adopción en el Sistema Fiscal Colombiano (Bachelor's thesis, Universidad EAFIT).

Palau, J. (2017). La primera aplicación de la NIIF 3 (2008). Combinaciones de negocios: un estudio internacional (Doctoral dissertation).

Parker Randall Guatemala. (29 de junio de 2010). Aplicación en Guatemala de NIIF para pymes. Recuperado de: http://www.niifpymes.com/

Quijano, R. A., Ma, A., Alfredo, L., Medina Blum, F., \& Javier Fajardo, M. (2017). Cultura Organizacional En Mipymes Turisticas De Campeche, México. Revista Internacional Administracion \& Finanzas, 10(4), 91-102.

Ramírez, Á. Q. (2003). El aprendizaje en la empresa: la nueva ventaja competitiva. Educación y educadores, 6, 127-139. 
Ramos, K. J. (2014). Sistema de índices para la valoración de los activos intangibles. Contribuciones a la Economía. Vol. julio.

Rojas González, Y. M. (2017). La sustentabilidad empresarial en un entorno organizacional (Bachelor's thesis, Universidad de Bogotá Jorge Tadeo Lozano).

Romero, J. P. P., \& Zilber, M. A. (2015). Innovación en el sector cementero de Colombia: estudio de caso Cementos Tequendama. Estudios Gerenciales, 31(135), 171-182.

Ron, R., \& Sacoto, V. (2015). Las PYMES ecuatorianas: su impacto en el empleo como contribución del PIB PYMES al PIB total. Revista Espacios, 15.

Rovira, S., \& Hiriart, C. (2014). Innovación sustentable: espacios para mejorar la competitividad de las pymes argentinas.

Ruiz, C. M., González, V., \& Alba Cabañas, M. (2018). El comportamiento organizacional y las Normas Internacionales de Información Financiera: experiencia en una PYME. Apuntes Contables, (21).

Sánchez, D. (2014). Mecanismos para estructurar los niveles de financiamiento y endeudamiento para una Pyme en Ecuador, Sector Comercio (Bachelor's thesis).

Simarro, J. D., Tonelli, O. E., \& Ribalaygua, L. C. (2012). Gestión de intangibles en pymes turísticas: Un caso de aplicación de las directrices MERITUM. Estudios y perspectivas en turismo, 21(1), 249-269.

Solleiro, J. L., \& Castañón, R. (2005). Competitividad y sistemas de innovación: los retos para la inserción de México en el contexto global. Revista Iberoamericana, 5(15), 165-197.

Suárez, A. (2013). Sustentabilidad empresarial, seguridad energética y ética ambiental en Chile. Acta bioethica, 19(2), 199-208.

Suárez, E., Franquiz, N., M., \& Sarduy, A. (2018). Procedimiento contable para valorar y reconocer activos intangibles de propiedad intelectual en empresas estatales cubanas. Cofin Habana, 12(1), 147-163.

Superintendencia de Compañías Valores y Seguros (SUPERCIAS). (2019). Instructivo para la aplicación de las normas NIIF. Recuperado de: https://portal.supercias.gob.ec/wps/portal/Inicio/Inicio/SectorSocietario/Normativa/Re soluciones.

Valencia, V. (2012). Revisión documental en el proceso de investigación. Universidad Tecnológica de Pereira. Bogotá, Colombia.

Vásquez, M. G., \& Vergara, D. J. (2011). Rol del banco de Loja y cooperativa de ahorro y crédito Catamayo; en los procesos de centralización del capital en los sectores agricultura, ganadería, caza, pesca y silvicultura; explotación de minas y canteras; industrias manufactureras; y suministros de electricidad y agua, del cantón Catamayo (Bachelor's thesis). 
Velázquez, A. L. V., \& Vargas, J. G. (2013). La sustentabilidad como modelo de desarrollo responsable y competitivo. Ingeniería de Recursos Naturales y del Ambiente, 11, 97107.

Viteri, J. R. (2015). Gestión de la producción con enfoque sistémico. Quito: Universidad Tecnológica Equinoccial.

Zulueta, A., Asencio, J., Leyva, D., \& Montero, J. M. (2013). Sustentabilidad empresarial de proyectos mineros: el análisis multicriterio como perspectiva acertada para su evaluación. Minería y Geología, 29(4), 79-94. 


\section{PARA CITAR EL ARTÍCULO INDEXADO}

Llerena Gómez, J. M., Mayorga Díaz, M. P., López Jara, A. A., \& López Paredes, M. A. (2020). Los activos intangibles y la ventaja competitiva sustentable en las Pymes ecuatorianas. Visionario Digital, 4(3), 62-80. https://doi.org/10.33262/visionariodigital.v4i3.1236

\section{Liencia}

El artículo que se publica es de exclusiva responsabilidad de los autores y no necesariamente reflejan el pensamiento de la Revista Visionario Digital.

El artículo queda en propiedad de la revista y, por tanto, su publicación parcial y/o total en otro medio tiene que ser autorizado por el director de la Revista Visionario Digital.
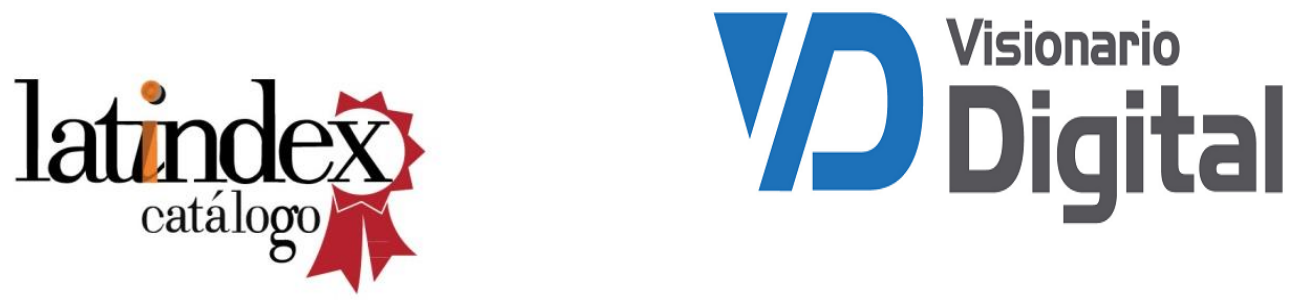